\title{
Solitary transverse colon metastasis: A rare case of uterine cervical cancer recurrence
}

\author{
Tatsuya Fukami*, Hiroshi Tsujioka, Sakiko Matsuoka, Sumire Sorano, Atsushi Tohyama, Hiroko \\ Yamamoto, Sumie Nakamura, Maki Goto, Ryoei Matsuoka, Fuyuki Eguchi
}

Department of Obstetrics and Gynecology, ASO Iizuka Hospital, Fukuoka, Japan

\begin{abstract}
A 44-years-old, Japanese woman underwent radical hysterectomy, preoperative chemotherapy and postoperative radiotherapy for uterine cervical cancer. She complained an abdominal palpable mass. The resection of transverse colon was performed. The postoperative pathological diagnosis was metastasis of uterine cancer without peritoneal dissemination. There have been few reports of metastatic colon cancer and most of them were gastric or lung cancer. We report a rare case of squamous cell carcinoma of uterine cervix metastatic to transverse colon.
\end{abstract}

Key Words: Cervical cancer, metastasis, transverse colon

\section{Introduction}

Cervical cancer is locally invasive, spreading primarily through direct extension. Advanced stages of cervical cancer is mainly metastases to lungs, liver, spleen, brain, and bone. We report a case of squamous cell carcinoma of the cervix metastatic to the solitary transverse colon causing palpable mass which can mimic primarily colon cancer. Because transverse colon metastases is rare, this case was initially thought to be of independent colon cancer, highlighting the importance of awareness of this site of metastases.

\section{Case report}

A 44-years-old gravida 3, para 3, Japanese woman complained with atypical genital bleeding. She was diagnosed squamous cell carcinoma by uterine cervix biopsy. The diagnosis of cervical carcinoma with bulky mass $(80 \times 77 \mathrm{~mm})$ and extension to the left side parametrium, but not reached to the pelvic wall (FIGO stage 2B). Preoperative chemotherapy as neoadjuvant chemotherapy was undertaken with a combination anti-cancer drug (CDDP $60 \mathrm{mg} / \mathrm{sqm}$, CPT-11 $60 \mathrm{mg} / \mathrm{sqm}$ ). The chemotherapy was very effective and the bulky mass was 99\% reduced on magnetic resonance imaging (MRI). She was performed laparotomy. The intraabdominal finding revealed no evidence of macroscopic peritoneum dissemination. The tumor was shrinked by preoperative chemotherapy and we found the defect of posterior uterine wall which seemed to be original tumor mass (Figure 1a). The left iliac and inguinal lymph nodes were swollen. Finally, we achieved radical hysterectomy operation. Pathological diagnosis revealed nonkeratinizing squamous cell carcinoma with mild vascular invasion at original uterine tumor (Figure 1b) and left iliac lymph node metastasis. No evidence of invasion into parametrium and vaginal wall was found (pT2BN1M0). She was irradiated $(51 \mathrm{~Gy})$ as adjuvant therapy and the radiation therapy was finished, there is no evidence of disease and tumor marker (SCC) decreased to normal level.

She had a subjective symptom of abdominal mass around navel after the end of 3 months of radiation therapy and tumor marker (SCC) elevated. She was checked up by computerized tomography (CT) and positron emission tomography (PET). They revealed $2 \mathrm{~cm}$ mass below the abdominal wall around navel both in CT (Figure 2a) and PET-CT (Figure 2b) compared with previous check-up. Exploratory abdominal operation, revealed no local recurrence and peritoneum dissemination, however, revealed nodular $2 \mathrm{~cm}$ palpable mass in the middle portion of the transverse colon. Macroscopic finding shows the gross appearance of the mucosa surface

*Corresponding Author: Tatsuya Fukami, Department of Obstetrics and Gynecology, ASO Iizuka Hospital 3-83 Yoshio-machi, Iizuka-city, Fukuoka 820-8505, JAPAN Phone: +81-948-22-3800, Fax: + 81-948-29-5744, E-mail: fukami1975@msn.com 

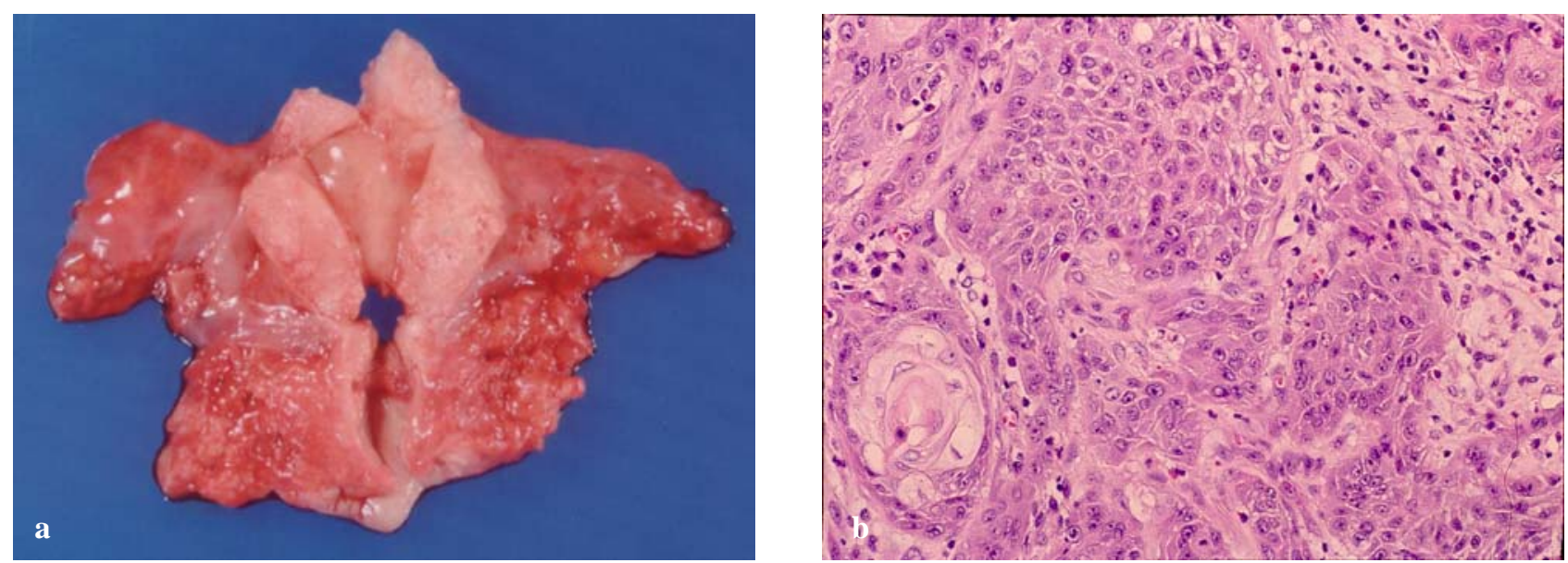

Fig. 1. Pathological findings (a) Macroscopic findings of uterus shows defect of posterior uterine wall by preoperative chemotherapy. (b) Histological findings shows non-keratinizing squamous cell. (Hematoxylin and eosin stain; $\mathrm{x}$ 400).
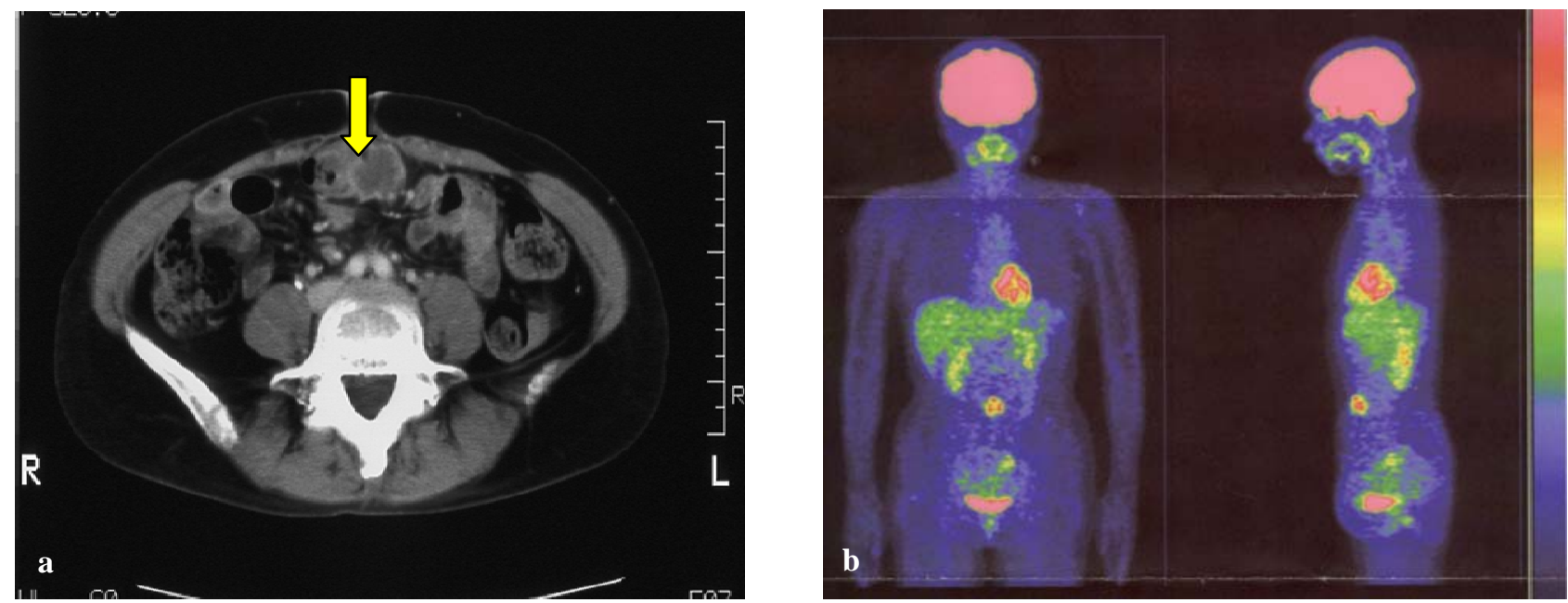

Fig. 2. PET-CT and CT images. Recurrent mass below the abdominal wall around navel (arrow). (a) CT, (b) PETCT.

in small intestinal tumor (Figure 3a). The mass was completely excised with transverse colon $(15 \mathrm{~cm})$. Findings of final pathology revealed nonkeratinizing squamous cell carcinoma (Figure 3b) invaded into the myometrium, but mucosa surfaces are normal in appearance (Figure 3c). Washing cytology was negative. Two months later, the patient was found with new lesions (liver metastasis) after $2^{\text {nd }}$ line chemotherapy, but her condition was deteriorated and died on disease 1 year later.

\section{Discussion}

The solitary metastasis of bowel is quite rare in uterine cervical cancer. The most frequently cause is results of direct extension or diffuse peritoneal disseminations from original malignant tumor (1). The metastatic tumor was no invasion of the mucosal surface of the small intestine lesions and consistent with the initial histologic findings of primary cervical cancer. We excluded primary small intestine cancer. The metastatic tumor was no invasion of the serous surface of the small intestine lesions and localized in the muscle layer. Finally, metastatic pattern of this case is vascular transition rather than peritoneal dissemination because it showed non-keratinizing squamous cell carcinoma with mild vascular invasion both in metastatic intestinal tumor and original uterine tumor. Gastric cancer $(60.5 \%)$ and lung cancer $(28.0 \%)$ are main causes of solitary metastasis to be bowel through hematogeneous or lymphogeneous metastasis (2). Cervical cancer tends to metastasize to regional and para-aortic lymph nodes before spreading to distant organs. The symptom in this case was a palpable mass. It is reported that symptoms as rectal pain, anorexia, 

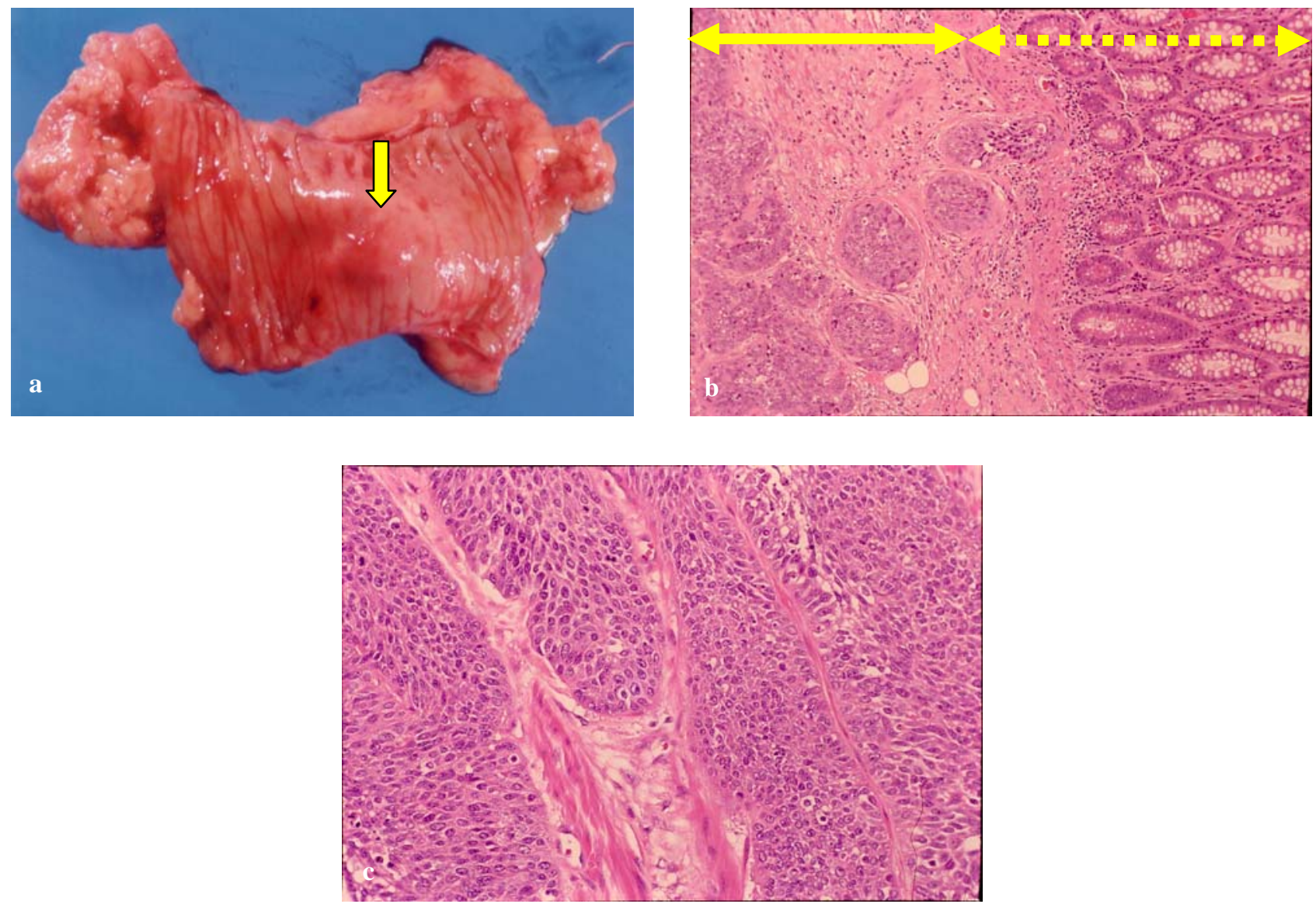

Fig. 3. Recurrent intestinal tumor. (a) Macroscopic finding shows the gross appearance of the mucosa surface in small intestinal tumor $(2 \times 2 \mathrm{~cm})$. Arrow indicates tumor. (b) Histological findings shows non-keratinizing squamous cell carcinoma. Solid line region indicates myometrium. Dotted line region indicates mucosa. (Hematoxylin and eosin stain; x100). (c) Histological findings shows non-keratinizing squamous cell carcinoma invaded into the myometrium, but mucosal surfaces are normal in appearance. (Hematoxylin and eosin stain; $\mathrm{x} 400$ ).

weight loss and a palpable mass (3). It is often difficult to diagnose of recurrence in patients following radiotherapy for cervical cancer. Signs and symptoms of early recurrent disease are often lacking and complains are attributed to the sideeffects of radiotherapy. The treatment for the localized metastatic lesion should be surgical extirpation. But, the survival rate of five years is $12.5 \%$ that is reported in the bowel recurrent cases (3).

Our case showed a solitary metastasis to the transverse colon without any evidence of recurrence, which is a rare case in patients of cervical cancer. The precise mechanism of tumor development in this case remain uncertain but metastatic pattern of this case is vascular transition rather than peritoneal dissemination because it showed non-keratinizing squamous cell carcinoma with mild vascular invasion both in metastatic intestinal tumor and original uterine tumor.

Conflict of Interests: The author declares that there is no conflict of interests regarding the publication of this paper.

\section{References}

1. Kodama J, Hongo A, Mizutani Y, et al.. A rare case of solitary metastasis of cervical cancer to the colon after radiation therapy. Eur J Gynaecol Oncol 1999; 20: 281-282.

2. Meyers MA and McSweeney J. Secondary neoplasms of the bowel. Radiology 1972; 105: 111.

3. McMahon AJ. Squamous carcinoma of the colon: primary or metastatic? Eur J Surg Oncol 1991; 17: 397-402. 